\title{
N6-Methyladenosine Modification of PTTG3P Contributes to Colorectal Cancer Progression via IGF2BP2
}

\author{
yang zheng \\ liaoning cancer hospital \\ guilin yu \\ Liaoning Cancer Institute and Hospital \\ longfei xie \\ University of California Berkeley \\ yue wang \\ Liaoning Cancer Institute and Hospital \\ guohua zhao ( $\nabla$ glimmerbar@163.com ) \\ Liaoning Cancer Institute and Hospital
}

\section{Research Article}

Keywords: Colorectal carcinoma, long non-coding RNA, PTTG3P, m6A, IGF2BP2

Posted Date: February 11th, 2021

DOI: https://doi.org/10.21203/rs.3.rs-205276/v1

License: (c) (1) This work is licensed under a Creative Commons Attribution 4.0 International License. Read Full License 


\section{Abstract}

Background N6-methyladenosine $\left(\mathrm{m}^{6} \mathrm{~A}\right)$ and long noncoding RNAs (IncRNAs) emerged as crucial players in colorectal cancer (CRC) progression, but the $\mathrm{m}^{6} \mathrm{~A}$ modified IncRNA PTTG3P in CRC are still need to be systematically defined. Methods qRT-PCR was adopted to measure the PTTG3P expression. Survival analysis was used to explore the correlation between the expression of PTTG3P and CRC patients prognosis. Receiver operating curve (ROC) was tested to evaluate the PTTG3P predictive ability. Functional studies were examined by CCK-8, glucose uptake, lactate assay, ATP assay, ECAR assay and xenograft mice model. Mechanistic studies were explored by GSEA, methylated RNA immunoprecipitation sequencing (MeRIP-Seq) and RNA immunoprecipitation (RIP).

Results PTTG3P was upregulated in CRC and closely related to poor prognosis. Through gain and loss of function approaches, PTTG3P facilitated proliferation and glycolysis through Hippo pathway, and glycolysis inhibitor (2-DG ,3-BG) and LDHA knockdown could rescue cell proliferation. Mechanically, $\mathrm{m}^{6} \mathrm{~A}$ methylation induced the elevation of PTTG3P by increasing its stability, and insulin like growth factor-2 mRNA binding proteins 2 (IGF2BP2) involved in the progression. Finally, rescue assays validated the effect of METTL3/PTTG3P/YAP1 axis in CRC progression.

Conclusions $\mathrm{m}^{6} \mathrm{~A}$-induced PTTG3P could facilitates $\mathrm{CRC}$ development via interacting with IGF2BP2, which provides a predictive biomarker and theraperutic target for CRC.

\section{Background}

Colorectal cancer (CRC) remains one of the major triggers of deaths from malignant tumors. Globally more than 1 million people suffer CRC every year ${ }^{[1]}$. As of $2012, C R C$ is the fourth cause of cancer death after lung, stomach, and liver cancer ${ }^{[2]}$. Despite improvements in diagnosis and combined treatment, patients with CRC have an even worse prognosis, especially in advance patients. Therefore, it is quite urgent to clarify the mechanism, even potential approaches for the therapeutic intervention of CRC.

Accumulating evidence has shown that pseudogene, a type of long noncoding RNA, exhibites pivotal functions. It is estimated that human genome has more than 18,000 pseudogenes. And pseudogene has emerged as key regulators of important biological processed involved in the development of human cancers. For instance, increased CYP4Z1 expression promotes tumor angiogenesis and growth in breast cancer partly via PI3K/Akt and ERK1/2 activation ${ }^{[3]}$. Nanog regulates primitive hematopoiesis by directly repressing critical erythroid lineage specifiers ${ }^{[4]}$. PTENP1 can exert a growth suppressive role by regulating cellular levels of PTEN ${ }^{[5]}$. LncRNA PTTG3P (pituitary tumor-transforming 3 , pseudogene, NR_002734), located at chromosome 8q13.1, was first reported in the study of human pituitary tumor transforming gene (hPTTG) family in $2000^{[6]}$. However, its biological function of Warburg effect has yet to be illustrated in CRC. N6-methyladenosine $\left(\mathrm{m}^{6} \mathrm{~A}\right)$ depicts the methylation of the nitrogen at position 6 in the adenosine base within mRNA. N6-Methyladenosine $\left(\mathrm{m}^{6} \mathrm{~A}\right)$ was originally characterised in the 
$1970 \mathrm{~s}^{[7]}$. Nowadays, the linkages between $\mathrm{m}^{6} \mathrm{~A}$ and malignant tumors have been indicated, including breast cancer, prostate cancer, pancreatic cancer, kidney cancer, eukaemia, stomach cancer, sarcoma, and leukaemia ${ }^{[8-12]}$.

Our data discovered that highly expressed PTTG3P, induced by METTL3, predicts poor prognosis in patients with CRC. Further study revealed that PTTG3P could facilitate proliferation and glycolysis by regulating METTL3/PTTG3P/YAP1 axis. This research might provide a prospective therapeutic target for CRC treatment.

\section{Materials And Methods}

Clinical samples

120 patients with CRC were enrolled from the liaoning cancer hospital and\#\#nubbetween March 2010 and November 2015. The including criteria were as follow: patients were with definite pathological diagnosis; No patients were received with chemotherapy or radiotherapy before surgery. The tumor and paired non-tumor tissues were also collected after lesion excision with $30 \mathrm{mins}$ and stored in liquid nitrogen, then transferred to $-80^{\circ} \mathrm{C}$ refrigerator. And the characteristics of cases were thoroughly noted. All of the CRC patients have signed informed consent prior to utilizing the clinical resources for investigation aims.The study was approved by the Ethics Committee of Liaoning cancer hospital.

Cell lines culture

Five human CRC cell lines (HT29, SW620,HCT-8, SW480 and HCT-116,) and normal human intestinal epithelial cell line (FHC, NCM460) were obtained from ATCC (Manassas, VA, USA). And cultured according to their instructions. All cells were cultured in an incubator according to their instructions at $37^{\circ} \mathrm{C}$ and in a humidified atmosphere with $5 \% \mathrm{CO} 2$.

Total RNA isolation, qRTPCR and transfection

The expression levels of RNA were calculated by the qRT-PCR system. Total RNA was extracted by TRIzol Reagent (Invitrogen), and $1 \mu \mathrm{g}$ of total RNA was reverse transcribed using the PrimeScript RT Reagent Kit (Perfect Real-Time; Takara). pcDNA3.1-PTTG3P, PTTG3P-containing lentiviral sequence vector (shPTTG3P) were purchased from GeneChem Corporation (Shanghai, China). CRC cells were transfected with plasmids in the presence of Lipofectamine 3000 (Invitrogen). After $48 \mathrm{~h}$ of transfection, cells were gathered for further use in following experiments. The gene expression quantity was calculated using the $2^{-\Delta \Delta C t}$ method.

Cell proliferation assay

Cell viability assay was carried out to analyze cell proliferation. Cell viability was estimated using CCK8 (CK04, DOJINDO, Beijing, China), on the basis of the manufacturer's instruction. Cells were seeded in 96- 
well culture plates. After incubation for indicated time, CCK-8 reagent $(10 \mu \mathrm{l})$ was added to each well.Cell viability was measured with a microplate reader for the absorbance at a wavelength of $450 \mathrm{~nm}$.

Flow cytometry of apoptosis

CRC cells in 6-well plates were rinsed in phosphate buffer saline (PBS), and then were trypsinized and resuspended in $100 \mu \mathrm{L}$ binding buffer added with $2.5 \mu \mathrm{L}$ of fluorescein isothiocyanate (FITC) conjugated Annexin $\mathrm{V}$ and $1 \mu \mathrm{L}$ of PI (Invitrogen). Fifteen minutes later, flow cytometry (BD Biosciences) was utilized for apoptotic cells.

Glucose, lactate, adenosine triphosphate (ATP) levels, and extracellular acidification rate (ECAR)

The levels of glucose and lactate were calculated with a Glucose Colorimetric Assay Kit (BioVision,CA) and a Lactate Assay Kit (BioVision, CA) in line with the instructions of manufacturer. ATP level was tested using Cell Titer-Glo Luminescent Cell Viability Assay (Promega, Madison, MI). ECAR was detected using Seahorse XF 96 Extracellular Flux Analyzer (Agilent Technologies, Santa Clara, CA) according to the manufacturer's instructions.

$\mathrm{m}^{6} \mathrm{~A}$ analysis

The quantification of $\mathrm{m}^{6} \mathrm{~A}$ RNA methylation level in total RNA was detected using the $\mathrm{m}^{6} \mathrm{~A}$ RNA methylation detection kit (Epigentek, Farmingdale, NY), according to the manufacturer's instructions.

Methylated RNA immuneprecipitation (MeRIP) assay

Total RNA was extracted from cells using TRIzol (Invitrogen) following the manufacturer's instructions. $\mathrm{m}^{6} \mathrm{~A}$ antibody (Abcam) and Magna methylated RNA immune-precipitation (MeRIP) $\mathrm{m}^{6}$ AKit (Merck Millipore) was explored to immunoprecipitate chemically fragmented RNA ( 100 nucleotides) according to its instruction. Enrichment of $\mathrm{m}^{6} \mathrm{~A}$ containing RNA was measured by qRT-PCR.

Animal study

HCT-116 cells were transfected with sh-PTTG3P. $1 \times 10^{7}$ indicated cells were subcutaneously injected into 4-week-old male nude mice. Tumor volume measured every 5 days. After 35 days, the mice were sacrificed, and the tumor weight was measured. The animal study was carried out in accordance with the Guide for the Care and Use of Laboratory Animals of the NIH. This study had been approved by the Committee on the Ethics of Animal Experiments of Baise hospital.

\section{Statistical analysis}

All the data were showed as the mean \pm standard deviation, at least three independent experiments. Data were compared using the chi-square ( $\chi 2$ test), Student's t-test or one-way analysis of variance (ANOVA). ROC curve was applied to evaluate the predictive ability of CRC diagnosis. Univariate and multivariate 
analysis were performed to ascertain independent factors for the diagnosis of CRC. Kaplan-Meier method was applied to measure the overall survival (OS). SPSS 22.0 (SPSS Inc., Chicago, IL, USA) was used to conduct statistical analyses, and differences were ensured when P-value was $<0.05$.

\section{Results}

PTTG3P is highly expressed in CRC

To evaluate potential IncRNAs involved in mediating CRC progression, we examined the IncRNA expression profile (GSE 84983) (Figure S1a). Comparison between CRC tumor tissues and adjacent normal tissues, we focused on the upregulated IncRNAs (fold change $>5, P<0.01$ ), for these IncRNAs might be oncogenes and therapeutic targets. LncRNA PTTG3P was one of the most upregulated and chosen for consideration (Figure S1b). Then, we found that PTTG3P had rarely ability to code proteins, using the open-reading frames (ORFs) Finder and conserved domain database. Moreover, five other different online metrics got the same conclusion (Table S3). Additionally, we identified no valid Kozak consensus sequence in PTTG3P ${ }^{[13]}$, indicating that PTTG3P is a long noncoding RNA with no proteincoding potential.

To verify the elevation of PTTG3P in CRC, we investigated the detailed annotative process of preclinical human cancer models via the Cancer Cell Line Encyclopedia (CCLE) (www.broadinstitute.org/ccle), indicating that PTTG3P is remarkably overexpressed in cell lines of CRC (Fig. 1a, 1b). Then, the cell lines of HT29, HCT-8, SW480, HCT-116 and FHC (human normal colorectal mucosal cell) were conducted for PTTG3P expression. As showed in Fig. 1c, the PTTG3P expression was exceedingly increased in HT29, HCT-8, SW480 and HCT-116 cells, compared with FHC cells.

Further, we explored PTTG3P expression in a cohort of 60 paired and non-tumor tissues of CRC, the clinicopathologic characteristics are demonstrated in Table 1. Significantly, the PTTG3P level was overexpressed in CRC tissues compared to their counterparts (Fig. 1d, 1e), which was in accordance with the results of TCGA database (Fig. 1f, 1g). Besides, high PTTG3P expression was observed in many kinds of tumors compared with normal counterparts (Fig. 1h). Also, our specimens confirmed PTTG3P overexpression in stomach adenocarcinoma (STAD), and esophageal squamous cell carcinoma (ESCA) (Fig. 1i,1j). All together, these data revealed that PTTG3P was elevated in CRC and might be an oncogene. 
Table 1

Clinicopathologic characteristics of studied patients in CRC

\begin{tabular}{|c|c|}
\hline Characteristics & Number of cases (\%) \\
\hline \multicolumn{2}{|l|}{ Age (years) } \\
\hline$\leq 60$ & 520 \\
\hline$>60$ & 680 \\
\hline \multicolumn{2}{|l|}{ Gender } \\
\hline Male & 560 \\
\hline Female & 640 \\
\hline \multicolumn{2}{|l|}{ Tumor size (cm) } \\
\hline$<5$ & 810 \\
\hline$>5$ & 390 \\
\hline \multicolumn{2}{|c|}{ Tumor invasion depth } \\
\hline T1-2 & 950 \\
\hline T3-4 & 250 \\
\hline \multicolumn{2}{|c|}{ Lymph node metastasis } \\
\hline No & 400 \\
\hline $\mathrm{N} 1-2$ & 800 \\
\hline \multicolumn{2}{|l|}{ Vessel invasion } \\
\hline Yes & 650 \\
\hline No & 550 \\
\hline \multicolumn{2}{|c|}{ Distant metastasis } \\
\hline Yes & 115() \\
\hline No & 50 \\
\hline \multicolumn{2}{|l|}{ Differentiation } \\
\hline Well & 380 \\
\hline Moderate & 620 \\
\hline Poor & 200 \\
\hline
\end{tabular}

High PTTG3P level correlates with poor prognosis 
To identify the connection between the level of PTTG3P and clinicopathologic features, we divided the cases into PTTG3P low-expression and high-expression group on the basis of median expression.

Upregulated PTTG3P was positive linked with Tumor size $(P=0.02)$ and Differentiation $(P=0.01)$, but not with age $(P=0.86)$, gender $(P=0.74)$, tumor invasion depth $(P=0.28)$, lymph node metastasis $(p=0.09)$ or vessel invasion $(P=0.06$ ) (Table 2). Moreover, the PTTG3P expression was higher in stage III-IV (advanced stage) than stage I-II (early stage) in tissues (Fig. 2a). Additionally, Kaplan-Meier survival curves illustrated that patents with highly expressed PTTG3P had poorer survival time (Fig. 2b). Further, we determined the prognostic ability of PTTG3P in CRC. As shown in Table 3, univariate analyses suggested highly expressed PTTG3P was associated with a dramatic risk of death $(P<0.01)$. Multivariate analysis demonstrated that PTTG3P expression could be an independent prognostic factor $(P<0.01)$. Subsequently, ROC curve was carried out to evaluate the diagnostic value of PTTG3P in CRC tissues compared with normal counterparts, the area under the ROC curve (AUC) was 0.776 (95\% Cl $0.733-$ 0.819) (Fig. 2C). Thus, these data suggested that high expression of PTTG3P predicted a worse prognosis and may serve as a clinical biomarker for CRC patients. 
Table 2

Correlation between PTTG3P expression in serum and clinicopathologic characteristics of ovarian cancer patients

\begin{tabular}{|c|c|c|c|c|}
\hline \multirow[t]{2}{*}{ Variable } & \multicolumn{3}{|c|}{ PTTG3P expression } & \multirow[t]{2}{*}{ P-value } \\
\hline & Total $(n=120)$ & High expression & Low expression & \\
\hline Age (years) & & & & 0.86 \\
\hline$\leq 60$ & 52 & 27 & 26 & \\
\hline$>60$ & 68 & 32 & 35 & \\
\hline Gender & & & & 0.74 \\
\hline Male & 56 & 30 & 28 & \\
\hline Female & 64 & 29 & 33 & \\
\hline Tumor size (cm) & & & & 0.02 \\
\hline$\leq 5$ & 81 & 47 & 37 & \\
\hline$>5$ & 39 & 16 & 24 & \\
\hline Tumor invasion depth & & & & 0.28 \\
\hline T1-2 & 95 & 53 & 43 & \\
\hline T3-4 & 25 & 12 & 20 & \\
\hline Lymph node metastasis & & & & 0.09 \\
\hline No & 40 & 25 & 20 & \\
\hline N1-2 & 80 & 36 & 39 & \\
\hline Vessel invasion & 65 & 49 & 20 & 0.06 \\
\hline Yes & 55 & 20 & 31 & \\
\hline No & & & & \\
\hline Differentiation & & & & 0.01 \\
\hline Well & 38 & 20 & 18 & \\
\hline Moderate & 62 & 46 & 16 & \\
\hline Poor & 20 & 13 & 7 & \\
\hline
\end{tabular}


Table 3

Univariate and multivariate analyses of clinicopathologic characteristics for correlations with overall survival

\begin{tabular}{|c|c|c|c|c|}
\hline \multirow[t]{2}{*}{ Variables } & \multicolumn{2}{|l|}{ Univariate analysis } & \multicolumn{2}{|l|}{ Multivariate analysis } \\
\hline & $\mathrm{HR}(95 \% \mathrm{Cl})$ & $P$ value & $\mathrm{HR}(95 \% \mathrm{Cl})$ & $P$ value \\
\hline PTTG3P expression & $1.758(1.085-2.850)$ & $<0.01$ & $1.712(1.053-2.782)$ & $<0.01$ \\
\hline Tumor size & $1.650(1.086-2.508)$ & $<0.01$ & $1.923(1.276-2.898)$ & $<0.01$ \\
\hline Differentiation & $1.724(1.183-2.511)$ & $<0.01$ & $1.724(1.183-2.511)$ & $<0.01$ \\
\hline
\end{tabular}

PTTG3P promotes glycolysis and proliferation in CRC

To investigate the biological function of PTTG3P, we respectively transfected the PTTG3P overexpressed plasmids and silenced shRNA targeting PTTG3P into HT-29 and HCT-116 cells,respectilvely (Fig. 2d). By determining PTTG3P expression via gene set enrichment analysis (GSEA) the Cancer Genome Atlas (TCGA) profiles, we found that PTTG3P levels were positively correlated with the glycolysis by affecting genes in glycolysis regulation (Fig. 2e). To verify results of this analysis, PTTG3P knockdown restrained the mRNA level of GLUT-1, ALDOA, PKM2 and LDHA. Intriguingly, the effect of sh-PTTG3P on glycolytic gene transcription could be rescued by PTTG3P re-expression (Fig. 2f). Next, we performed the glucose uptake analysis, ATP analysis, lactate production analysis, and discovered that sh-PTTG3P repressed these. In contrast, PTTG3P overexpression boosted the glucose uptake (Fig. 3a), lactate production (Fig. 3b), and ATP accumulation (Fig. 3c). Additionally, we calculated the level of ECAR, sh-PTTG3P notably repressed glycolytic capacity and vice verse (Fig. 3d). Also, we found that silenced PTTG3P suppressed the proliferation and facilitated apoptosis of HCT-116 cells, whereas upregulated PTTG3P increased the proliferation and inhibited apoptosis of HT-29 cells according to the CCK-8 assay and flow cytometry analysis (Fig. 3e,3f). In vivo, highly expressed PTTG3P efficiently increased the tumor growth (Fig. 3g,3h). We then explored whether glycolysis plays a vital role in PTTG3P modulation of cell proliferation and tumor growth. Notably, the glycotic inhibitors 2-DG and 3-BP or depletion of LDHA, which could catalyze the final step of glycolysis, could partly abrogate cancer cell proliferation and tumor growth (Fig. 3i,3j,3k).

Clinically, oxaliplatin is used for treatment of colorectal cancer. Previously, it is reported that suppression of glycolysis is an effective strategy to block cell proliferation and conquer drug resistance. Hence, we speculated that PTTG3P ablation in combination with oxaliplatin could strikingly repress tumor growth. As shown in Fig. 3l,3m, PTTG3P depletion could be conducted simultaneously with oxaliplatin. As a taken, PTTG3P knockdown plus oxaliplatin is a promising therapy for CRC.

PTTG3P regulates Hippo signaling pathway in CRC 
In order to elucidate which pathway involved in PTTG3P-meddated CRC progression, GSEA in the published TCGA CRC database was explored. And we suggested that PTTG3P expression was associated with the YAP1-activated gene signatures, indicating that Hippo signaling pathway may participated in the biological function of PTTG3P (Fig. 4a). To verify the speculation, the hub genes in Hippo pathway, including LATS1/2, MST1/2 and YAP1, and Hippo pathway target genes, such as CDX2, FOXM1, CTGF and CYR61, were tested in sh-PTTG3P HCT-116 cells. Subsequently, PTTG3P knockdown impaired the mRNA level of YAP1, FOXM1 and CTGF (Fig. 4b).

It is commonly acknowledged that YAP1, a crucial factor in Hippo pathway, involves in cell proliferation and suppressed apoptotic genes. In our study, the level of PTTG3P and YAP1 displayed positive linkage in CRC tissues (Fig. 4c). And the association between YAP1 expression and clinicopathologic characteristics from TCGA indicated in table S4.

Besides, we performed rescue assays in HT-29 cells. PTTG3P overexpression plus YAP1 knockdown could reverse PTTG3P induced phenotype (Figure ,4d-4g). Intriguingly, the treatment of Hippo pathway inhibitor, XMU-MP-1 (inhibiting MST1/2), could not recover the promoting effect of PTTG3P on proliferation, apoptosis and tumor growth. (Fig. 4h-4k).In brief, all the data uncovered that PTTG3P hedges the key factor MST1/2, while modulates YAP1 in Hippo pathway to exhibit pivotal functions in CRC progression.

$\mathrm{m}^{6} \mathrm{~A}$ modification is involved in the overexpression of PTTG3P in CRC cells

We next explored the upstream factors for PTTG3P elevation in CRC. Our study found no influence on PTTG3P expression using DNA methyltransferase inhibition (Fig. 5a). Accumulating evidence has shown that ectopic expression of IncRNAs could be regulated by transcriptional factors, and histone acetylation plays a critical role in this procession. Then, we explored whether histone acetylation exerted a role in PTTG3P expression using SAHA and NaB, the broad-spectrum HDAC inhibitors, and we discovered that these HDAC inhibitors failed to alter PTTG3P level in HT29 cells (Fig. 5b). Further, overexpressed HDAC6 and HDAC8 had no effect on increasing PTTG3P expression (Fig. 5c). Subsequently, MeRIP-qPCR discovered that the $\mathrm{m}^{6} \mathrm{~A}$ modification expression was dramatically increasing in the CRC cells compared with normal cells (Fig. 5d). Then, we confirmed that METTL3, a writer of RNA modification, significantly elevated the PTTG3P expression in both HT-29 and HCT-116 cells (Fig. 5e). Besides, overexpressed ALKBH5, an eraser of RNA modification, greatly suppressed the PTTG3P expression (Fig. 5f). Moreover, we conducted RNA stability analysis by treating cells with Act-D, binding DNA at the initiation complex and preventing RNA chain elongation, our findings uncovered that highly expressed METTL3 strengthened the stability of PTTG3P (Fig. $5 \mathrm{~g}$ ). Therefore, $\mathrm{m}^{6} \mathrm{~A}$ modification acts as an crucial factor in PTTG3P expression. As recently reported, Insulin-like growth factor-2 mRNA-binding proteins 1, 2, and 3 (IGF2BP1-3) are described as a type of $\mathrm{m}^{6} \mathrm{~A}$ readers. We then evaluated the potential binding of PTTG3P and IGF2BP1-3, RIP-PCR was performed using an antibody against IGF2BP1-3. The results found that 
METTL3 overexpression increases binding between PTTG3P and IGF2BP2 in both HT-29 and HCT-116 cells (Fig. 5h). Interestingly, IGF2BP2 knockdown could partly abrogate the ability of Mettl3 upregulating PTTG3P (Fig. 5i). Finally, the association between METTL3,IGF2BP2 expression and clinicopathologic characteristics from TCGA indicated in table S5,S6.

The METTL3/PTTG3P/YAP1 axis facilitates the progression of CRC

To evaluate the role of METTL3/PTTG3P/YAP1 axis involved in proliferation and glycolysis, we perfomred a series of rescue experiments in HT-29 cells. Glucose uptake analysis, ATP analysis, lactate production analysis, and ECAR analysis showed that sh-METTL3 or sh-YAP1 repressed these phenomenon (Fig. 6a,6b,6c,6d). Whereas, sh-METTL3 plus overexpressed PTTG3P, or sh-YAP1 plus overexpressed PTTG3P could rescue these phenotype. In addition, the promotion of cancer cell proliferation could be counteracted by sh-PTTG3P plus overexpressed METTL3, or sh-YAP1 plus overexpressed PTTG3P (Fig. 6e,6f).

Hence, METTL3/PTTG3P/YAP1 axis conducted a pivotal role in CRC progression.

Additionally, METTL3/PTTG3P high and PTTG3P/YAP1 high group certified an unsatisfactory prognosis than low group (Fig. 6g,6h). We further found that higher levels of METTL3, ALKBH5, and IGF2BP2 predicted poor prognosis and diagnostic value in CRC (Figure S2).

\section{Discussion}

Pseudogene may be transcribed into RNA at low levels, due to promoter elements inherited from the ancestral gene or arising by new mutations. Although most of transcripts have rarely functional significance than chance transcripts from other parts of the genome, some have given rise to regulatory RNAs and new proteins. For instance, long noncoding RNA HK2P1, a pseudogene of HK2, promoted the lactate production and glucose uptake in endometrial stromal cells ${ }^{[14]}$. Pseudogene PTENP1 repressed the oncogenic PI3K/AKT pathway and inhibited the HCC progression ${ }^{[15]}$. To date, the role of pseudogene PTTG3P in glycolysis of CRC has not been reported yet. Our findings documented that PTTG3P facilitated CRC progression via a METTL3/PTTG3P/YAP1 axis.

Our study verified that PTTG3P is highly expressed and has a potential diagnostic value, with an AUC of 0.776 (95\% $\mathrm{Cl} 0.733-0.819)$ in CRC. Clinically, high PTTG3P expression considerably associate with tumor size and TNM stage as well as shorter survival time. These results confirmed that PTTG3P overexpression serves as a valuable prognostic biomarker and aids innovatively efficient therapies for CRC patients. Additionally, our findings stands in line with other research, Liu,et al. ${ }^{[16]}$ reported that PTTG3P was remarkably upregulated in CRC tumor samples than that in normal samples. Zhou, et al. ${ }^{[17]}$ 
revealed that PTTG3P is a valuable resource for identification in HCC progression and is useful for biomarker development. Weng, et al. ${ }^{[18]}$ certified that PTTG3P facilitates cell proliferation, migration and invasion and might serve as a new promising strategy for gastric cancer. Recently, PTTG3P expression has a relationship with breast cancer ${ }^{[19]}$ and pancreatic cancer ${ }^{[20]}$. Thus, the oncogenic role of PTTG3P in malignant tumors is strongly suggested.

Malignant tumors could undergo glycolysis at a higher speed than that of non-tumor tissue controls ${ }^{[21-}$ 23]. This phenomenon is known as Warburg effect ${ }^{[24]}$. The Warburg hypothesis demonstrates that malignant tumor is fundamentally caused by mitochondrial metabolism disorder. Doherty JR, et al. ${ }^{[23]}$ found that tumor lactate levels correlate with increased metastasis, tumor recurrence, and poor outcome. And targeting lactate metabolism is a prospective method for cancer therapeutics. Furthermore, cancer cells with high level of glycolysis and acid resistance have a energetic growth advantage, which facilitates unrestrained proliferation and invasion. In our study, we explored gain and loss-of-function approaches in HT29 and HCT-116 cells and found PTTG3P ablation resulted in the inhibition of CRC cell glycolysis by regulating numerous genes linked with metabolic pathways, whereas the opposite outcome was observed after enforced expression of PTTG3P. Nowadays, ketogenic diet was used to constrain glycolysis to starve cancer cells, adjusting mitochondrial metabolism ${ }^{[25]}$. Here, we also proposed that the biological mechanism of PTTG3P on boosting cell proliferation might resist to apoptosis.

Hippo signaling pathway has become increasingly important in human cancer ${ }^{26]}$, the key regulator YAP1 has been certified to be upregulated in breast cancer, colorectal cancer, and liver cancer ${ }^{[27]}$, and YAP1 could promote cell growth ${ }^{[2-30]}$ and inhibit apoptosis ${ }^{[31]}$. Clinically, YAP1 could be a target for the development of cancer drugs ${ }^{[32]}$. Yi, et al. ${ }^{[33]}$ suggested that inhibiting TEAD-YAP1 interactions or block the binding function of WW domains is a pharmacologically viable strategy against the YAP1 oncoprotein. In our presented study, we discovered that PTTG3P activates Hippo signaling pathway by promoting YPA1, FOXM1 and CTGF, not MST1/2, and rescue assay consolidates this by using Hippo pathway inhibitor, XMU-MP-1 (inhibiting MST1/2).

As recently reported, many studies have certified the important roles of METTL3 in the RNA degradation and stability. METTL3 ( $\mathrm{m}^{6} \mathrm{~A}$ methyltransferase $)$ and ALKBH5 $\left(\mathrm{m}^{6} \mathrm{~A}\right.$ demethylases) coordinately mediate the $m^{6} A$ modification of $m R N A$ level of $C D C P 1$, and $m^{6} A$ reader $Y T H D F 1$ could identify the $m^{6} A$ residues on CPCP1 mRNA 3'-UTR, which is installed by METTL3 to stimulate the CDCP1 translation ${ }^{[34]}$. METTL3 deficiency causes cancer cell apoptosis and repress

cancer cell invasion ${ }^{[35]}$, while the activation of ALKBH5 by hypoxia was found to induce cancer stem cell enrichment ${ }^{[36]}$. Our findings demonstrated that $\mathrm{m}^{6} \mathrm{~A}$ methylation definitely take part in PTTG3P upregulation, by enhancing its transcript stability. Moreover, We revealed that overexpressed METTL3 could enhance the binding between IGF2BP2 and PTTG3P, because of $\mathrm{m}^{6} \mathrm{~A}$-induced RNA structure alteration. Interestingly, IGF2BP2 depletion could partly abrogate the ability of Mettl3 upregulating PTTG3P. 


\section{Conclusion}

In conclusion, our study first discovers that METTL3/PTTG3P/YAP1 axis promotes Warburg effect in $\mathrm{CRC}$, and $\mathrm{m}^{6} \mathrm{~A}$ readers IGF2BP2 participates this progress. Hence, PTTG3P abalation might be used as a significant target for CRC prevention and therapy, shedding some light on the poorly understanding of $\mathrm{m}^{6} \mathrm{~A}$ and IncRNA in cancer biology.

\section{Abbreviations}

CRC: colorectal cancer; ROC: Receiver operating curve; MeRIP-Seq: methylated RNA immunoprecipitation sequencing; IGF2BP2:insulin like growth factor-2 mRNA binding proteins 2; hPTTG: human pituitary tumor transforming gene; ANOVA: one-way analysis of variance; OS: overall survival; PTTG3P: Pituitary tumor-transforming 3; IncRNA: long non-coding RNA;

\section{Declarations}

\section{Data Availability}

The datasets used and analyzed in the current study are available from the corresponding author on reasonable request.

Competing Interests

The authors declare that there are no competing interests associated with the manuscript.

Funding

Supported by Natural Science Foundation of Liaoning Province of China.

Author Contributions

The work presented here was carried out in collaboration between all authors. Yue wang and guohua zhao contributed to the conception of the study; yang zheng contributed significantly to analysis and manuscript preparation. Guilin yu performed the data analyses and wrote the manuscript; longfei xie helped perform the analysis with constructive discussions.

\section{Statement of Ethics}

Patients have given their written informed consent in our study. And ethics committee of the first hospital of dandong and liaoning cancer hospital approved the study protocol.

Consent to Publication

All the authors agreed to publish the manuscript. 
Acknowledgement

We appreciate all the participants who provide supports for our research.

\section{References}

1. Cunningham D, Atkin W, Lenz HJ. et al. Colorectal cancer. Lancet.2010, 375 (9719): 1030-1047.

2. Adelstein BA, Macaskill P, Chan SF. et al. Most bowel cancer symptoms do not indicate colorectal cancer and polyps: a systematic review. BMC Gastroenterology. 2011;11:65.

3. Rieger MA. et al. Identifification of a novel mammary-restricted cytochrome P450, CYP4Z1, with overexpression in breast carcinoma. Cancer Res.2004, 64, 2357-2364.

4. Wei Yu H, Chai Y, Li. et al.Increased expression of CYP4Z1 promotes tumor angiogenesis and growth in human breast cancer. Toxicol Appl Pharmacol. 2012;264(1):73-83.

5. Poliseno L, Salmena L, Zhang J. et al. A coding-independent function of gene and pseudogene mRNAs regulates tumour biology. Nature. 2010;24(7301):1033-8.. „; 465.

6. Chen L, Puri R, Lefkowitz EJ, Kakar SS. Identification of the human pituitary tumor transforming gene (hPTTG) family: molecular structure, expression, and chromosomal localization. Gene. 2000;248:4150 .

7. Lavi S, Shatkin AJ. Methylated simian virus 40-specific RNA from nuclei and cytoplasm of infected BSC-1 cells. Proc Natl Acad Sci USA. 1975;72:2012-6.

8. Reddy SM, Sadim M, Li J. et al. Clinical and genetic predictors of weight gain in patients diagnosed with breast cancer. Br J Cancer. 2013;20(4):872-81.. „109.

9. Stram DO, Tjønneland A, Travis R. et al. Association of type 2 diabetes susceptibility variants with advanced prostate cancer risk in the Breast and Prostate Cancer Cohort Consortium. Am J Epidemiol. 2012;176(12):1121-9.

10. Lin Y, Ueda J, Yagyu K. et al. Association between variations in the fat mass and obesity-associated gene and pancreatic cancer risk: a case-control study in Japan. BMC Cancer. 2013;13:337.

11. Casalegno-Garduño $R$, Schmitt A, Wang $X$, et al. Wilms' tumor 1 as a novel target for immunotherapy of leukemia. Transplantation Proceedings. 2010,42(8):3309-3311.

12. Linnebacher $M$, Wienck $A$, Boeck I. et al. Identification of an MSI-H tumor-specific cytotoxic $T$ cell epitope generated by the (-1) frame of U79260(FTO). J Biomed Biotechnol. 2010;2010:841451.

13. Kozak M. Point mutations define a sequence flanking the AUG initiator codon that modulates translation by eukaryotic ribosomes. Cell. 1986;44:283-92.

14. Lv H, Tong J, Yang J. et al. Dysregulated Pseudogene HK2P1 May Contribute to Preeclampsia as a Competing Endogenous RNA for Hexokinase 2 by Impairing Decidualization. Hypertension. 2018;71(4):648-58.

15. C-LC 1, Y-WT 1, J-C Wu, et al. Suppression of hepatocellular carcinoma by baculovirus-mediated expression of long non-coding RNA PTENP1 and MicroRNA regulation. Biomaterials. 2015,44:71-81. 
16. Liu N, Dou L, Zhang X. LncRNA PTTG3P Sponge Absorbs microRNA-155-5P to Promote Metastasis of Colorectal Cancer. 2020,(9)13:5283-5291.

17. Zhou Q, Zhang W, Wang Z. et al. Long non-coding RNA PTTG3P functions as an oncogene by sponging miR-383 and up-regulating CCND1 and PARP2 in hepatocellular carcinoma. BMC Cancer. 2019;24(1):731. ., ; 19.

18. Weng W, Ni S, Wang Y. et al. PTTG3P promotes gastric tumour cell proliferation and invasion and is an indicator of poor prognosis. J Cell Mol Med. 2017;21(12):3360-71.

19. Lou W, Ding B, Fan W. High Expression of Pseudogene PTTG3P Indicates a Poor Prognosis in Human Breast Cancer. Mol Ther Oncolytics. 2019;27:14:15-26.

20. Liu W, Tang J, Zhang H. et al. A novel IncRNA PTTG3P/miR-132/212-3p/FoxM1 feedback loop facilitates tumorigenesis and metastasis of pancreatic cancer. Cell Death Discov. 2020;30(1):136. . „6.

21. GATENBY R A, GILLIES RJ. Why do cancers have high aerobic glycolysis? [J]. Nat Rev Cancer. 2004;4(11):891-9.

22. OU J, MIAO H, MA Y. et al. Loss of abhd5 promotes colorectal tumor development and progression by inducing aerobic glycolysis and epithelial-mesenchymal transition. Cell Rep. 2014;9(5):1798 - 811.

23. CLEVELAND DOHERTYJR. J L. Targeting lactate metabolism for cancer therapeutics. J Clin Invest. 2013;123(9):3685-92.

24. WARBURG 0. On the origin of cancer cells. Science. 1956;123(3191):309 - 14.

25. SCHWARTZ L, SEYFRIED T, ALFAROUK K O. et al. Out of Warburg effect: An effective cancer treatment targeting the tumor specific metabolism and dysregulated pH [J]. Semin Cancer Biol, 2017, 43134-138.

26. Saucedo LJ, Edgar BA. "Filling out the Hippo pathway". Nature Reviews Molecular Cell Biology. August 2007;8(8):613-21.

27. Zender $L$, Spector MS, Xue $W$,et al. Identification and validation of oncogenes in liver cancer using an integrative oncogenomic approach.Cell. 2006,30; 125(7): 1253-1267.

28. Shimomura T, Miyamura N, Hata S. et al. The PDZ-binding motif of Yes-associated protein is required for its co-activation of TEAD-mediated CTGF transcription and oncogenic cell transforming activity. Biochem Biophys Res Commun. 2014;443(3):917-23.

29. Harvey KF, Zhang X, Thomas DM. The Hippo pathway and human cancer. Nature Reviews Cancer. 2013;13(4):246-57.

30. Johnson R, Halder G. The two faces of Hippo: targeting the Hippo pathway for regenerative medicine and cancer treatment. Nature Reviews Drug Discovery. 2014;13(1):63-79.

31. Zhao B, Kim J, Ye X. et al. Both TEAD-binding and WW domains are required for the growth stimulation and oncogenic transformation activity of yes-associated protein. Can Res. 2009;69(3):1089-98. 
32. Sudol M, Shields DC, Farooq A. Structures of YAP protein domains reveal promising targets for development of new cancer drugs. Semin Cell Dev Biol. 2012;23(7):827-33.

33. Liu-Chittenden Y, Huang B, Shim JS. et al. Genetic and pharmacological disruption of the TEAD-YAP complex suppresses the oncogenic activity of YAP. Genes Dev. 2012;26(12):1300-5.

34. Fan Yang $H$, Jin B, Que. et al. Dynamic m(6)A mRNA methylation reveals the role of METTL3-m(6)ACDCP1 signaling axis in chemical carcinogenesis. Oncogene. 2019;38(24):4755-72.

35. Shuibin Lin J, Choe P, Du,et al. The m(6)A Methyltransferase METTL3 Promotes Translation in Human Cancer Cells.2016,5;62(3):335-345.

36. Zhang C, Samanta D, Lu H. et al. Hypoxia induces the breast cancer stem cell phenotype by HIFdependent and ALKBH5-mediated mA-demethylation of NANOG mRNA. Proc Natl Acad Sci U S A. 2016;5(14):E2047-56. ., ;13.

\section{Figures}

A
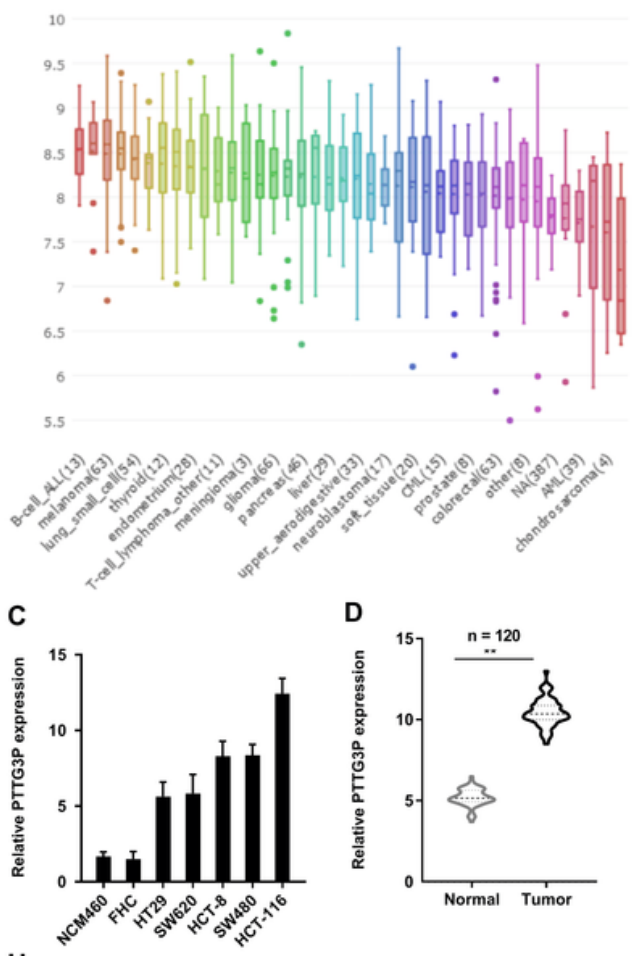

H

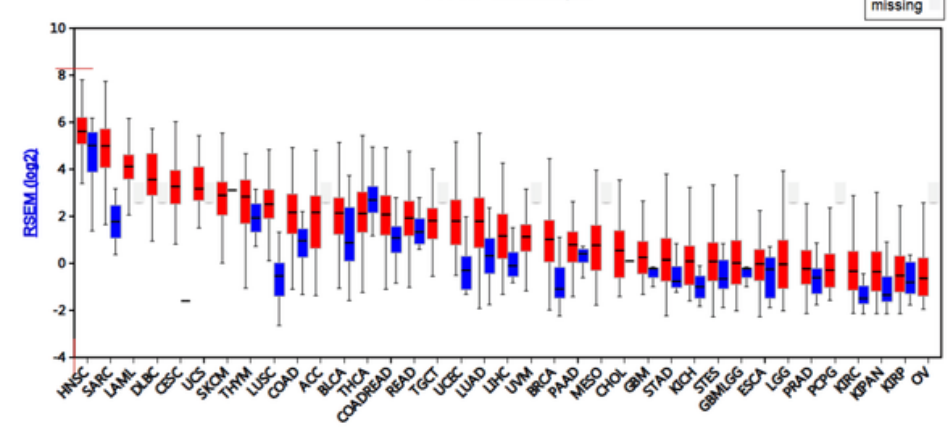

E
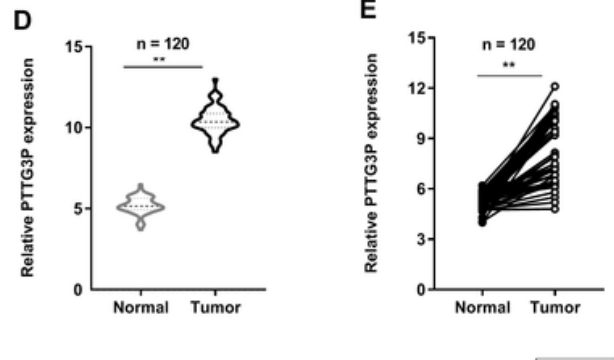

B
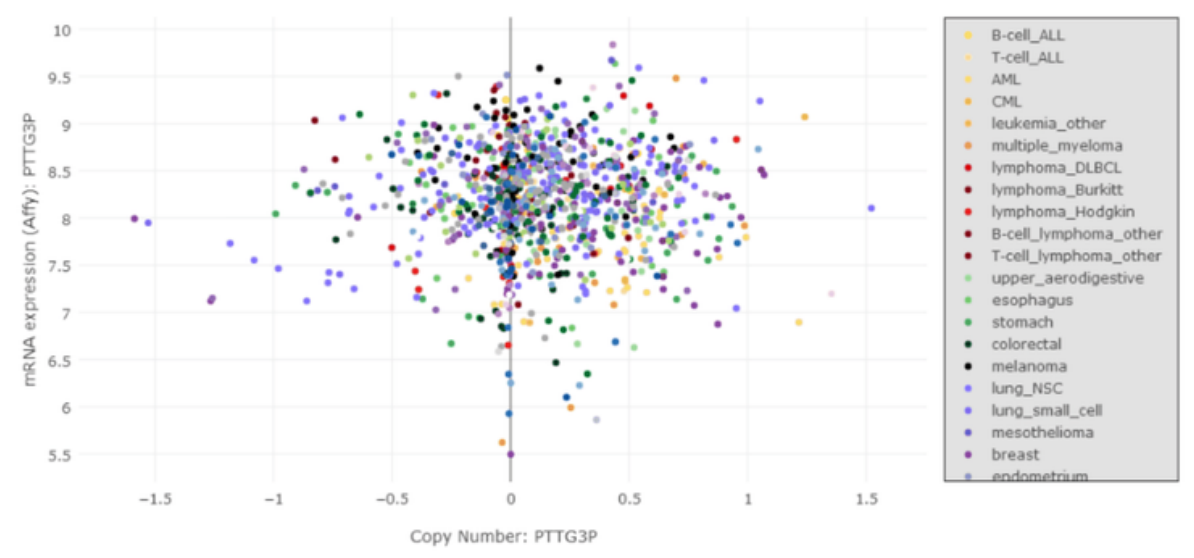

F TCGA-COAD $(n=521)$

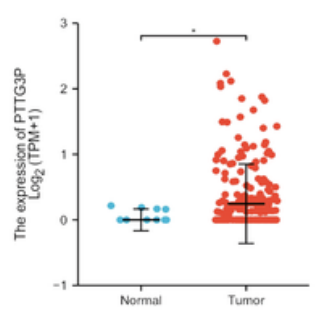

G
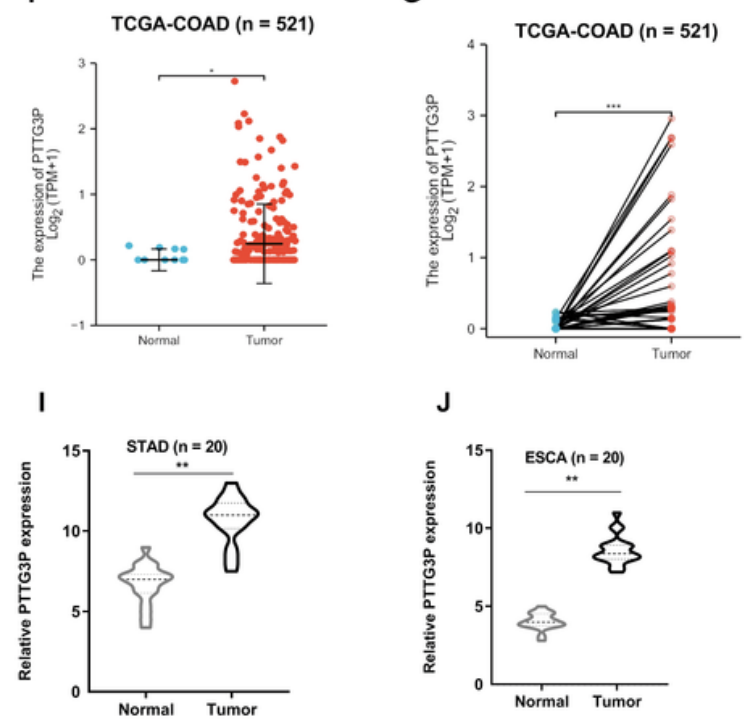

J

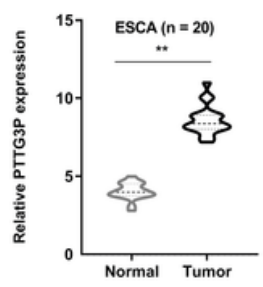




\section{Figure 1}

PTTG3P is upregulated in CRC tissues, and cell lines. (A,B) Exploring PTTG3P expression in NSCLC cell lines by assembling the Cancer Cell Line Encyclopedia (CCLE) (www.broadinstitute.org/ccle). (C) The expression profiles of PTTG3P in HT29, HCT-8, SW480, HCT-116 and FHC was detected with qRT-PCR. $(\mathrm{D}, \mathrm{E})$ qRT-PCR analysis was used to evaluate PTTG3P expression in 120 paired tumor and paired adjacent non-tumor tissues. $(F, G)$ High PTTG3P expression was observed in TCGA database of CRC. $(H)$ High PTTG3P expression was observed in many kinds of tumors. $(\mathrm{I}, \mathrm{J})$ High PTTG3P expression was observed in STAD and ESCA. *P $<0.05,{ }^{\star *} \mathrm{P}<0.01$, ${ }^{\star \star \star} \mathrm{P}<0.001$ 
A

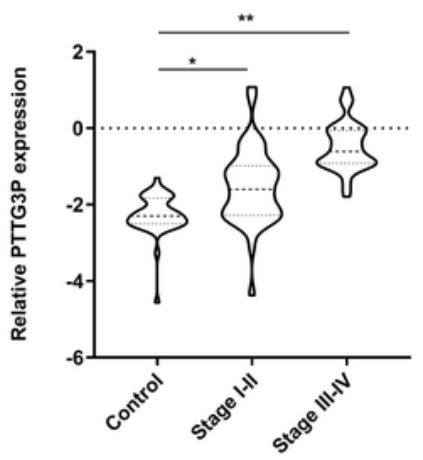

C

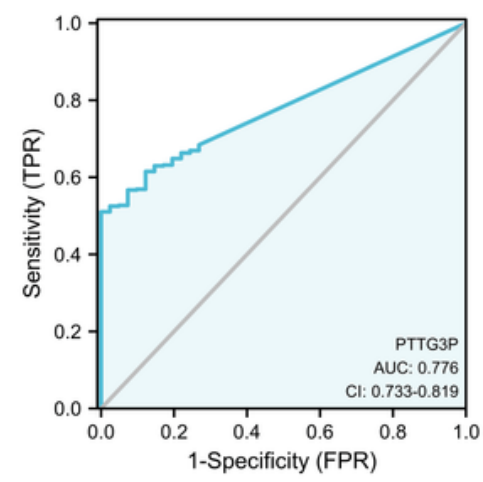

E

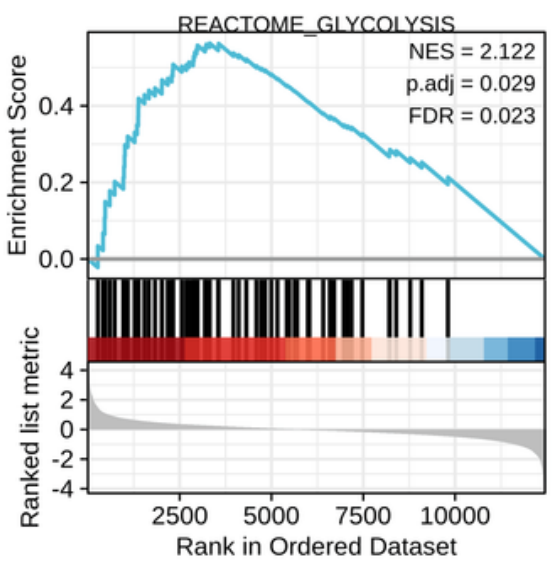

B

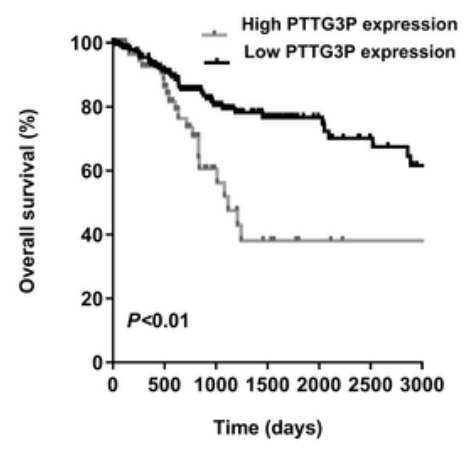

D
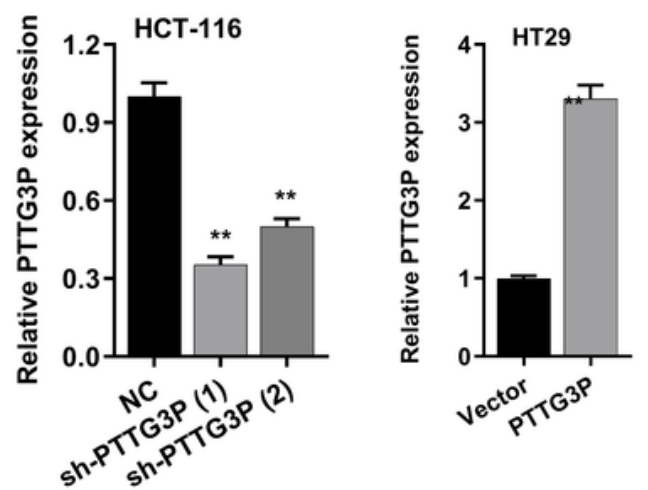

$\mathbf{F}$

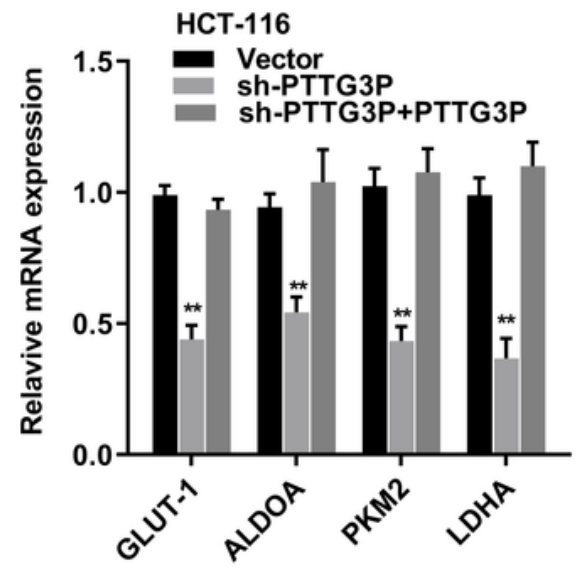

Figure 2

Overexpressed PTTG3P correlates with poor prognosis in CRC. (A) Comparison of the expression level of PTTG3P in serum among healthy controls, early stage RCC patients, and late stage RCC patients. (B) PTTG3P expression and survival predicted poor prognosis of OS in a cohort of 120 paired cases. (C) ROC curve analysis of the diagnostic performance of serum PTTG3P (D) Short hairpin RNA (shRNA) targeting PTTG3P and PTTG3P overexpressed plasmids were transfected into HCT-116 and HT29 cells. qRT-PCR 
was used to evaluate the transfection efficiency. (E) GSEA plot showing that PTTG3P expression positively correlated with glycolysis-activated gene signatures (REACTOME GLYCOLYSIS). (F) Analysis of glycolytc gene expression in PTTG3P knockdown or PTTG3P knockdown A2780 cells transfected with PTTG3P. *P $<0.05, * * P<0.01, * \star * P<0.001$

A
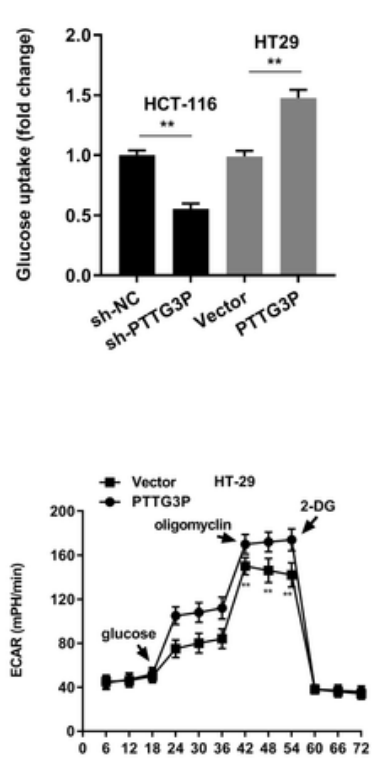

Time (mins)
B

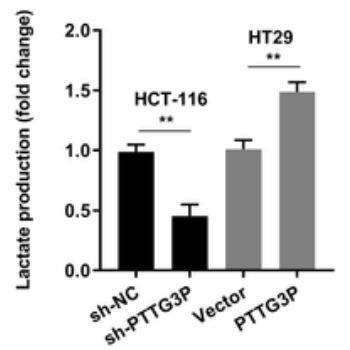

E

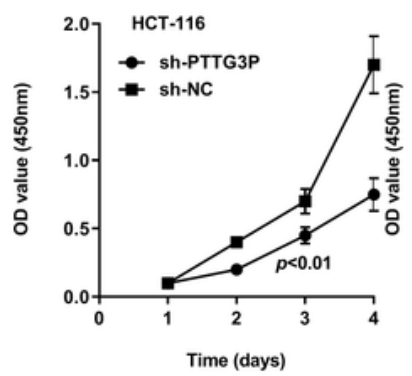

C

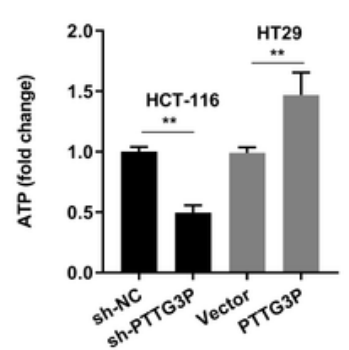

D

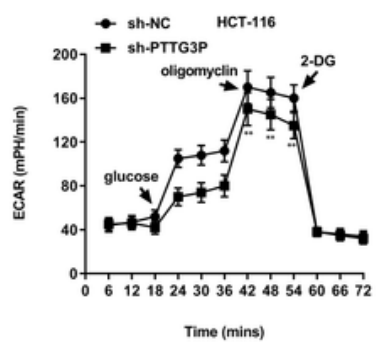

$\mathbf{F}$

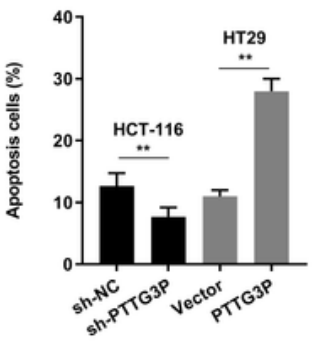

K

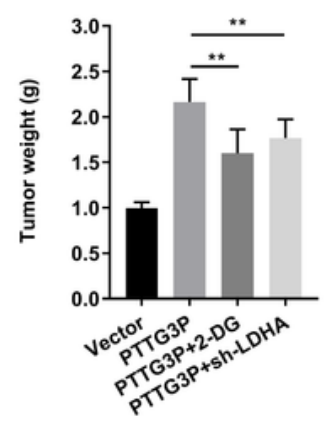

H

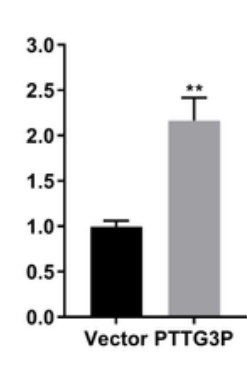

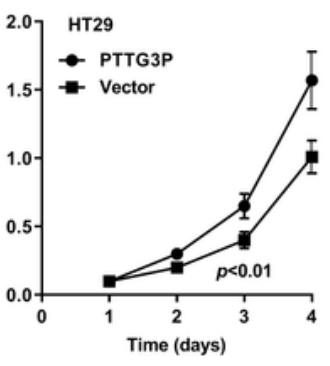

I
J

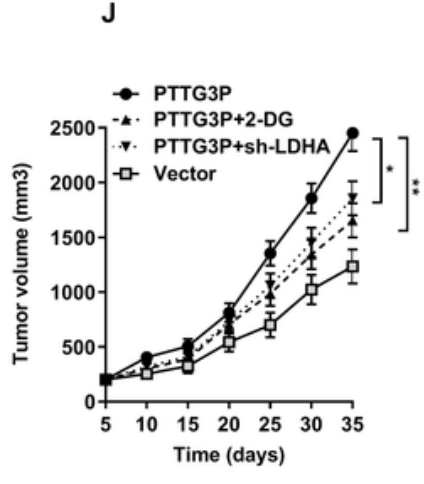

M

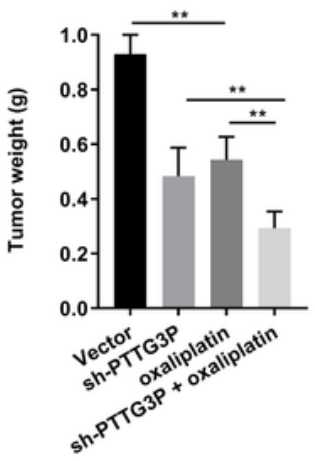

Figure 3 
PTTG3P promoted the glycolysis and proliferation of CRC. (A) Glucose uptake analysis, (B) Lactate production analysis, and (C) ATP analysis explored the glucose uptake, lactate production, and ATP accumulation. (D) Extracellular acidification rate (ECAR) analysis tested the glycolytic capacity in CRC cells transfectedwith sh-PTTG3P or overexpressed PTTG3P. (E) CCK8 assay detected the proliferation of HCT-116 and HT29 cells transfected with sh-PTTG3P or overexpressed PTTG3P. (F) Flow cytometry assays revealed that PTTG3P affected cell apoptosis $(\mathrm{G})$ Tumor volume and $(\mathrm{H})$ weight were measured in vivo when injected with sh-PTTG3P transfected HCT-116 cells. (I) CCK8 assay detected the proliferation of HT29 cells transfected with overexpressed PTTG3P and treated with 2.5mM 2-DG or $100 \mu \mathrm{M}$ 3-BP. (J) Xenograft tumors volume, $(K)$ Xenograft tumors weight were established, with injected with PTTG3P or PTTG3P plus sh-LDHA or PTTG3P treated with 2.5mM 2-DG HT29 cells . Empty vector as indicated. (L) Tumor volume and (M) weight were measured in vivo when injected with sh-PTTG3P and oxaliplatin treatment transfected HCT-116 cells. Data are presented as the mean \pm SD from three independent experiments. ${ }^{*} P<0.05, * \star P<0.01, \star \star * P<0.001$ 
A

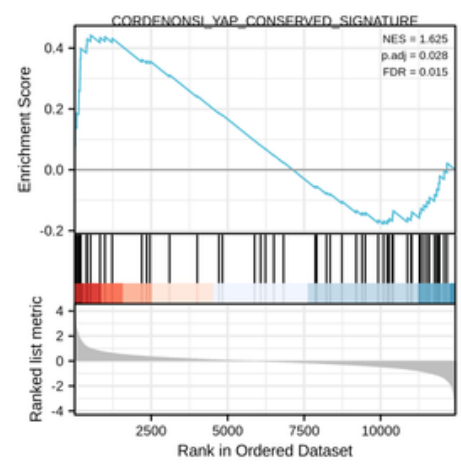

D

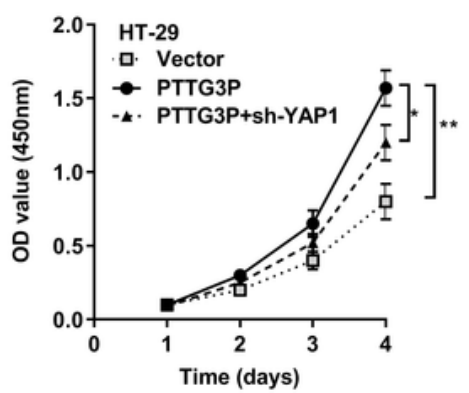

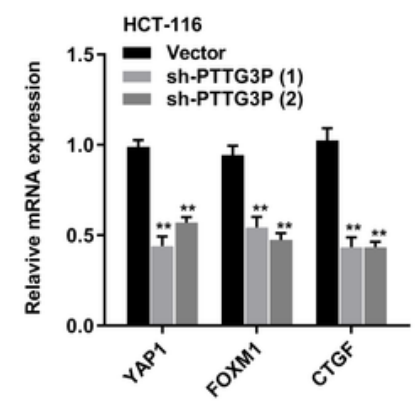

E

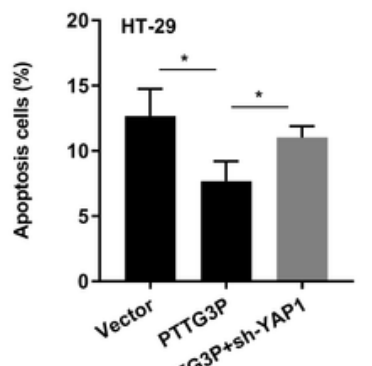

H

G

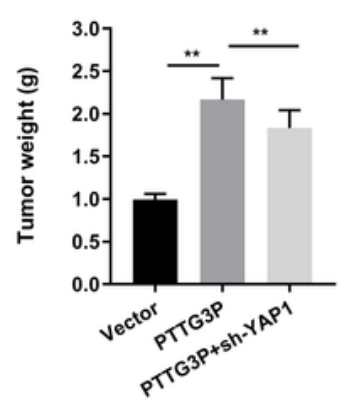

J

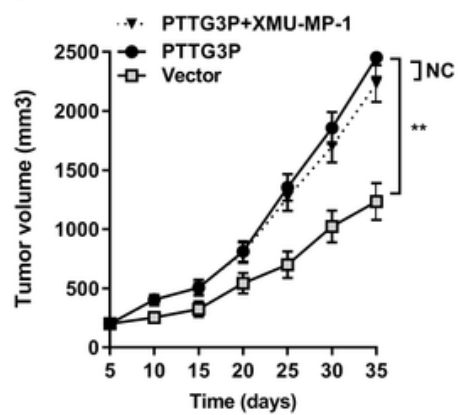

K
C

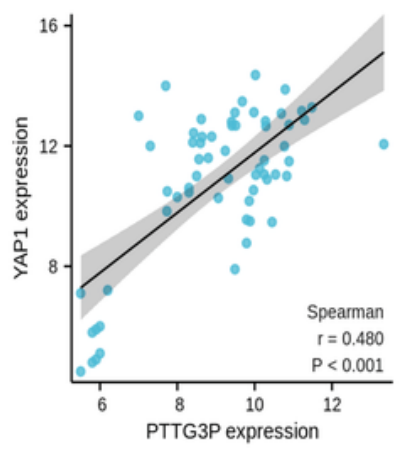

F

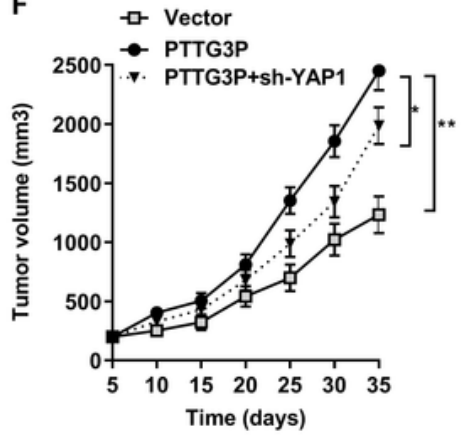

I

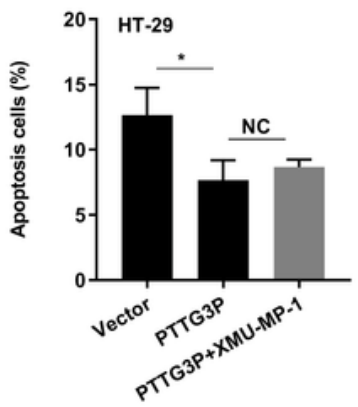

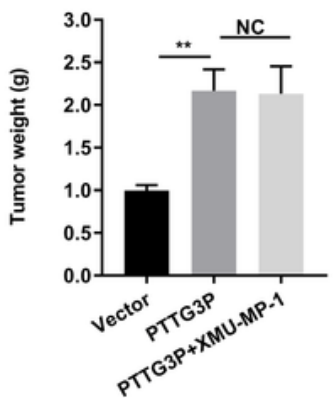

\section{Figure 4}

PTTG3P regulates Hippo signaling pathway in CRC (A) GSEA plot showing that PTTG3P expression positively correlated with YAP-activated gene signatures (B) PTTG3P knockdown impaired the mRNA level of YAP1, FOXM1 and CTGF. (C) The relationship between PTTG3P and YAP1 was analysed by Spearman's correlation analysis. (D) CCK8 assay detected the proliferation of HT29 cells transfected with PTTG3P or PTTG3P plus sh-YAP1. (E) Flow cytometry assays revealed that PTTG3P plus sh-YAP1could 
partly rescue cell apoptosis. (F)Xenograft tumors volume, (G) Xenograft tumors weight were established, with injected with PTTG3P or PTTG3P plus sh-YAP1. Empty vector as indicated. (H) CCK8 assay detected the proliferation of HT29 cells transfected with PTTG3P or PTTG3P plus XMU-MP-1 (I) Flow cytometry assays revealed that PTTG3P plus XMU-MP-1could partly rescue cell apoptosis. (J)Xenograft tumors volume, $(K)$ Xenograft tumors weight were established, with injected with PTTG3P or PTTG3P plus XMUMP-1. Empty vector as indicated. Data are presented as the mean \pm SD from three independent experiments. ${ }^{*} \mathrm{P}<0.05,{ }^{*} \mathrm{P}<0.01,{ }^{* \star *} \mathrm{P}<0.001$

A

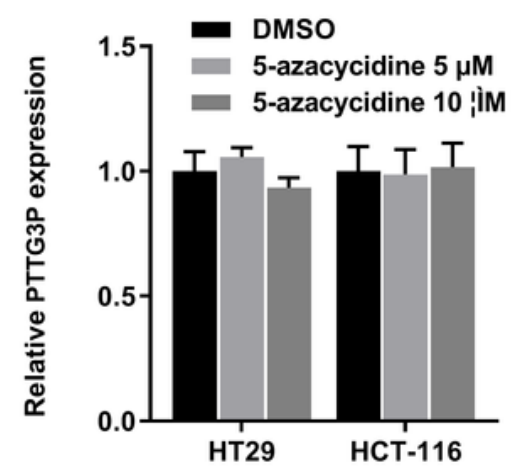

D

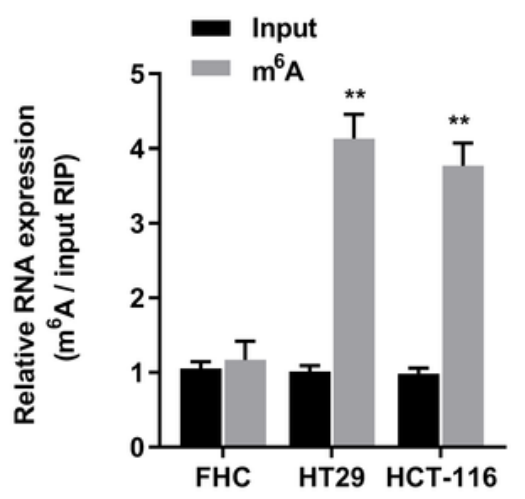

G

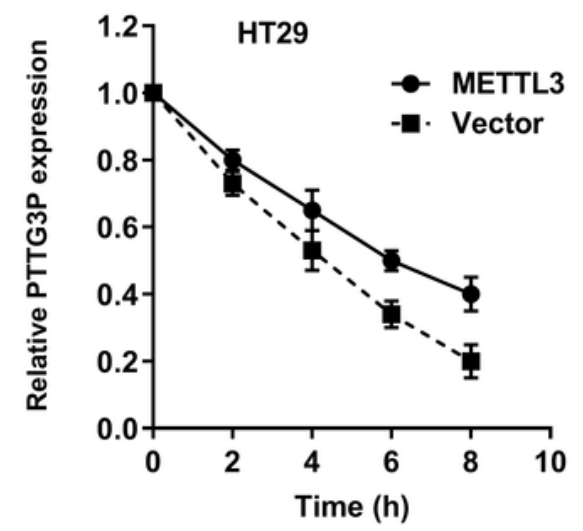

B

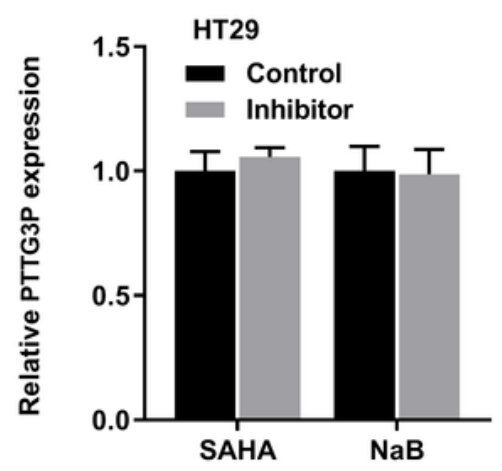

E

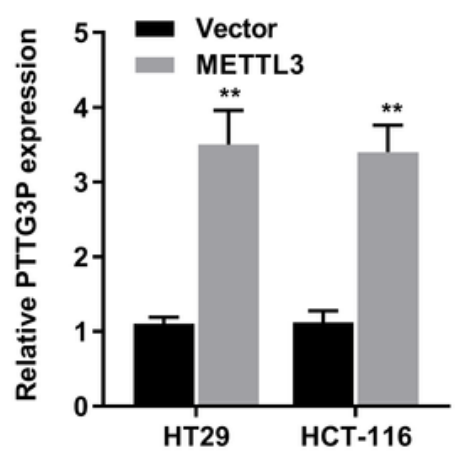

H

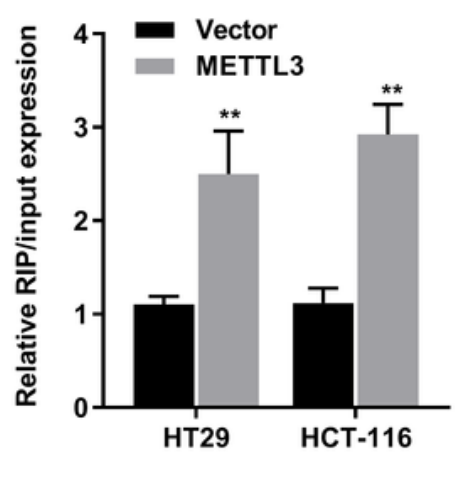

C

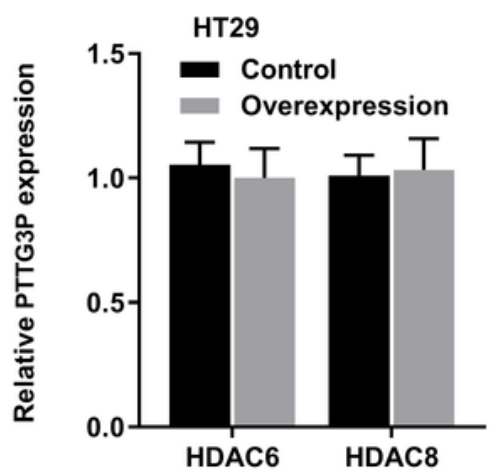

F

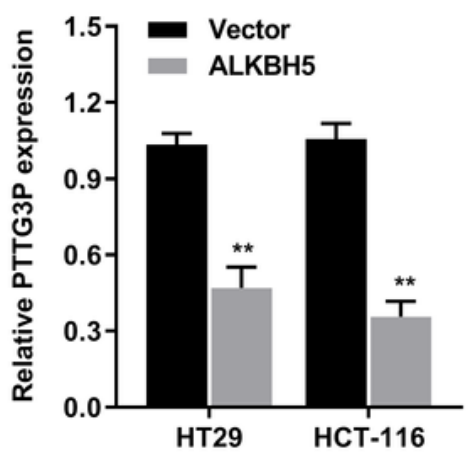

I

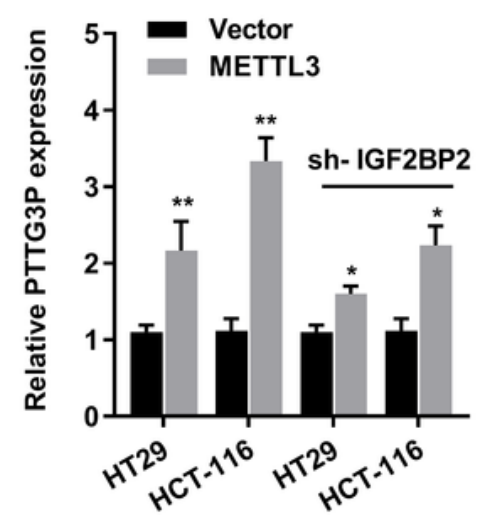




\section{Figure 5}

The m6A modification is involved in the ectopic expression of PTTG3P in CRC (A) qRT-PCR analysis of PTTG3P in A2780 cells treated with DMSO or 5-Azacytidine (5 $\mu \mathrm{M}$ or $10 \mu \mathrm{M})$ for $72 \mathrm{hr}(\mathrm{n}=3)$. (B) HT29 cells were treated with SAHA $(2 \mu \mathrm{M})$, or $\mathrm{NaB}(2 \mathrm{mM})$ for $24 \mathrm{~h}$, and RP11 mRNA was measured. (C) After transfection with vector control, pcDNA/HDAC6, or pcDNA/HDAC8 for $24 \mathrm{~h}$, PTTG3P expression in HCT15 cells was measured by qRT-PCR. (D) MeRIP-qPCR showed the m6A modification expression in HNOE cells as compared with the HT29 and HCT-116 cells. (E) The qRT-PCR analysis of PTTG3P levels in control and METTL3 overexpression in HT29 and HCT-116 cells. (F) The qRT-PCR analysis of PTTG3P levels in control and ALKBH5 overexpression in HT29 and HCT-116 cells. (G) RNA stability analysis found the stability of PTTG3P in A2780 cells treated with actinomycin D (Act-D, $5 \mu \mathrm{g} / \mathrm{m}$ ). (H) After transfection with vector control or Mettl3 for $24 \mathrm{~h}$, binding between PTTG3P and IGF2BP2 was analysed by RIP-PCRin HT29 and HCT-116 cells . (I) After transfection with vector control and sh-IGF2BP2 or METTLI3 and shIGF2BP2 for $24 \mathrm{~h}$, binding between PTTG3P and IGF2BP2 was analysed by RIP-PCRin HT29 and HCT-116 cells . Data are presented as the mean \pm SD from three independent experiments. ${ }^{*} p<0.05,{ }^{\star \star} p<0.01$ compared with control 
A

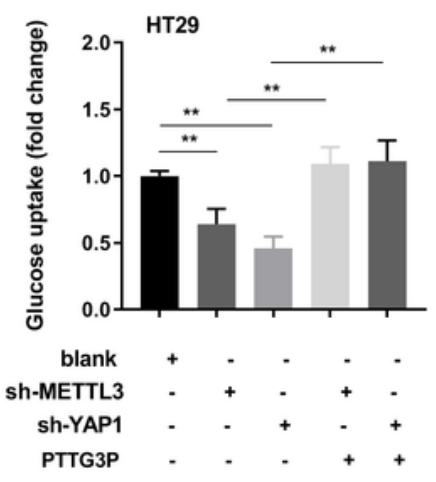

C
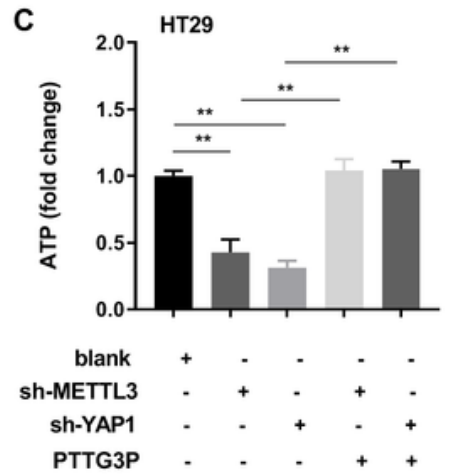

E

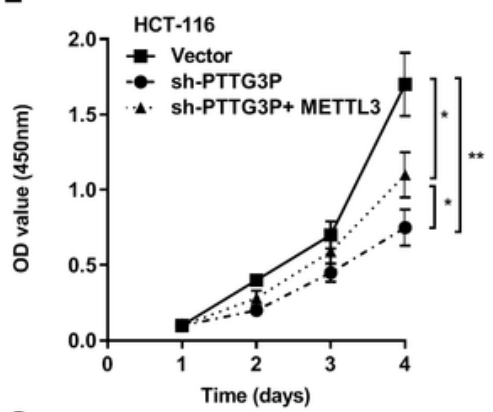

G

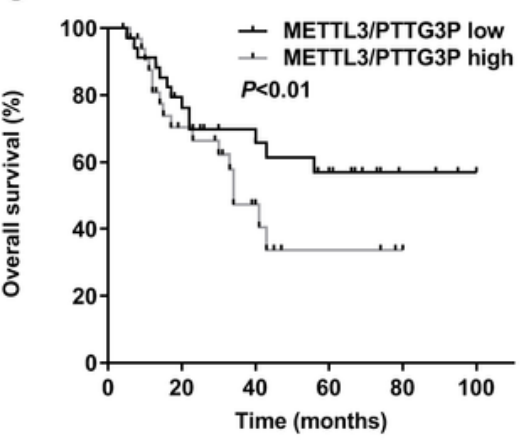

B

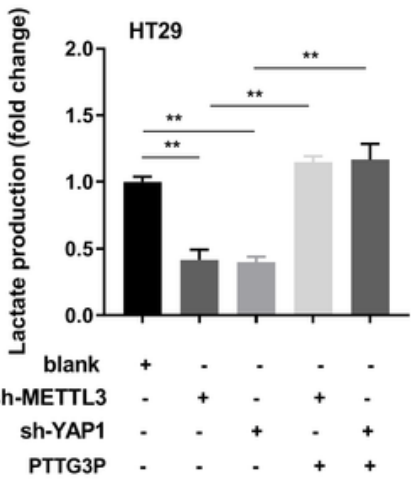

D
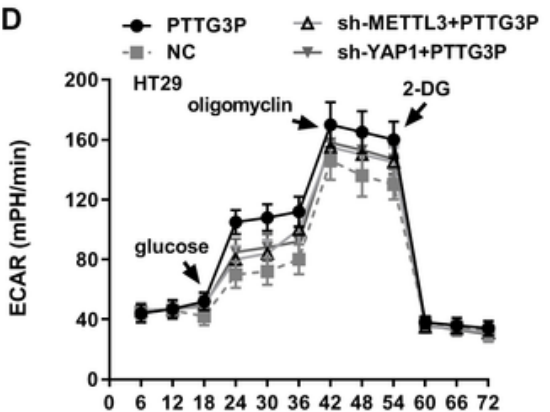

Time (mins)

$\mathbf{F}$

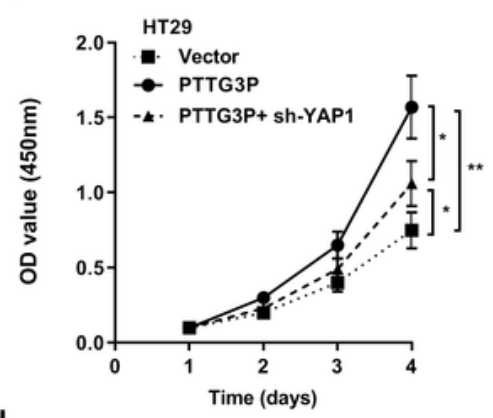

H

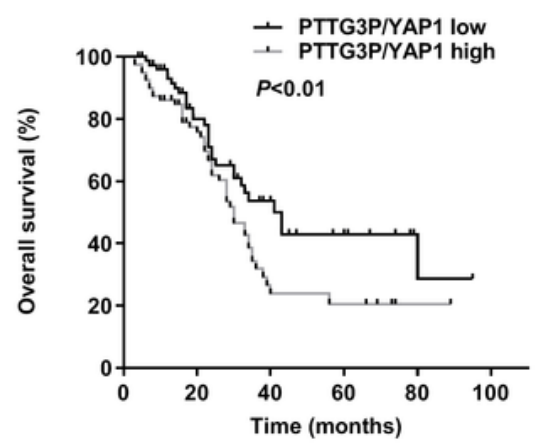

Figure 6

METTL3/PTTG3P/YAP1 axis axis facilitates proliferation and glycolysis in CRC (A) Glucose uptake analysis, (B) Lactate production analysis, (C) ATP analysis, and (D) extracellular acidification rate (ECAR) analysis were respectively carried out in $\mathrm{HT} 29$ cells to detect the glucose uptake, lactate production, ATP accumulation, and glycolytic capacity. HT29 cells were cotransfected with sh-METTL3 plus overexpressed PTTG3P, or sh-YAP1 plus overexpressed PTTG3P. (E) CCK8 assay detected the 
proliferation of HCT-116 cells transfected with sh-PTTG3P or sh-PTTG3P+METTL3. (F) CCK8 assay detected the proliferation of HT29 cells transfected with PTTG3P or PTTG3P+sh-YAP1. (G) Kaplan-Meier analysis of the OS curves for patients with METTL3/PTTG3P-high (both levels of METTL3/PTTG3P were high), METTL3/PTTG3P-low (both levels of METTL3/PTTG3P were low) expression. (H) Kaplan-Meier analysis of the OS curves for patients with PTTG3P/YAP1-high (both levels of PTTG3P/YAP1 were high), PTTG3P/YAP1-low (both levels of PTTG3P/YAP1 were low) expression. Data are presented as the mean \pm SD from three independent experiments. ${ }^{*} P<0.05, * \star P<0.01, * \star * P<0.001$

\section{Supplementary Files}

This is a list of supplementary files associated with this preprint. Click to download.

- SupplementaryTable1.doc

- SupplementaryTable2.doc

- SupplementaryTable3.doc

- SupplementaryTable4.doc

- SupplementaryTable5.doc

- SupplementaryTable6.doc

- figures1.tif

- figures2.tif 